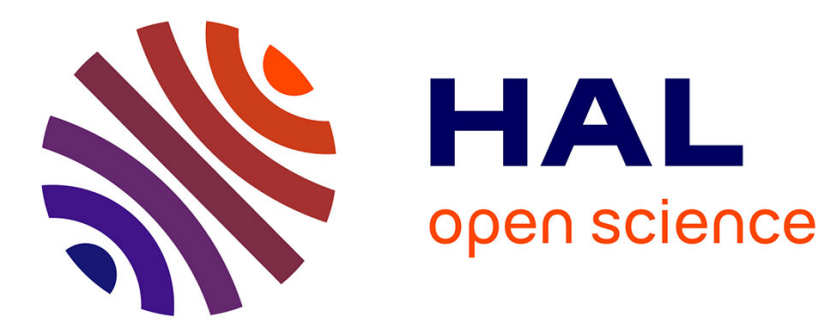

\title{
Multiple-view fluorescence optical tomography reconstruction using compression of experimental data
}

N. Ducros, A. Bassi, G. Valentini, M. Schweiger, S. Arridge, C. d'Andrea

\section{To cite this version:}

N. Ducros, A. Bassi, G. Valentini, M. Schweiger, S. Arridge, et al.. Multiple-view fluorescence optical tomography reconstruction using compression of experimental data. Optics Letters, 2011, 36 (8), pp.1377-1379. 10.1364/OL.36.001377 . hal-00873991

\section{HAL Id: hal-00873991 https://hal.science/hal-00873991}

Submitted on 16 Oct 2013

HAL is a multi-disciplinary open access archive for the deposit and dissemination of scientific research documents, whether they are published or not. The documents may come from teaching and research institutions in France or abroad, or from public or private research centers.
L'archive ouverte pluridisciplinaire HAL, est destinée au dépôt et à la diffusion de documents scientifiques de niveau recherche, publiés ou non, émanant des établissements d'enseignement et de recherche français ou étrangers, des laboratoires publics ou privés. 


\title{
Multiple-view fluorescence optical tomography reconstruction using compression of experimental data
}

\author{
Nicolas Ducros, ${ }^{1}$ Andrea Bassi, ${ }^{1}$ Gianluca Valentini,,${ }^{1,2}$ Martin Schweiger, ${ }^{3}$ \\ Simon Arridge, ${ }^{3}$ and Cosimo D'Andrea ${ }^{1,2, *}$ \\ ${ }^{1}$ Instituto di Fotonica e Nanotecnologie (IFN-CNR)—Dipartimento di Fisica, Politecnico di Milano, \\ Piazza Leonardo da Vinci 32, I-20133 Milan, Italy \\ ${ }^{2}$ Italian Institute of Technology (IIT), Piazza Leonardo da Vinci 32, I-20133 Milan, Italy \\ ${ }^{3}$ Centre for Medical Image Computing, University College London, Malet Place, London WC1E 6BT, UK \\ ${ }^{*}$ Corresponding author: cosimo.dandrea@polimi.it
}

Received January 6, 2011; revised March 12, 2011; accepted March 14, 2011; posted March 17, 2011 (Doc. ID 140742); published April 8, 2011

\begin{abstract}
We report on the experimental demonstration of a fast reconstruction method for multiview fluorescence diffuse optical tomography by using a wavelet-based data compression. We experimentally demonstrate that the use of data compression combined with the multiview approach makes it possible to perform a fast reconstruction of high quality. A structured illumination approach, guided by the compression scheme, has been adopted to further reduce the acquisition time. The reconstruction algorithm is based on the finite element method, and hence is suitable for samples of any arbitrary shape. (c) 2011 Optical Society of America
\end{abstract}

OCIS codes: $170.7050,170.3010,170.3880,110.0113$.

Optical molecular imaging is an emergent technique that aims at visualizing molecular processes in vivo and represents a fundamental tool for medical research by addressing the molecular bases of diseases [1]. In particular, fluorescence diffuse optical tomography (FDOT) aims at localizing and quantifying molecular specimens targeted by selective fluorescent markers. This makes it possible to perform longitudinal studies in small animals. FDOT is usually carried out by illuminating the animal with excitation light patterns (generally point sources) and collecting the fluorescence light on a portion of the animal's surface. By applying inverse algorithms, the distribution of the fluorochrome concentration within the sample is reconstructed [2]. Nevertheless, it is well known that the inverse problem is ill-posed. To face this challenge and to improve the spatial resolution, the emitted fluorescence is generally oversampled by means of a CCD detector (up to $10^{6}$ pixels) that captures the signal over a large area of the animal's surface. The measurements are repeated for many light source patterns, up to $10^{3}$ when a raster scanning is considered. This approach leads to a huge amount of data that requires a correspondingly very long processing time. This also leads to a long acquisition time, which may be incompatible with many in vivo experiments (e.g., due to the anaesthesia).

In the last few years, a novel approach based on data compression and/or structured illumination has been proposed, both in diffuse optical tomography [3-5] and FDOT [ $6-9$ ] , in order to drastically reduce the acquisition and reconstruction times, while preserving the information content. Data compression allows for reducing the computational effort required for reconstruction. The main idea behind this approach derives from the fact that tissue behaves like a low-pass filter for spatial Fourier components $[\underline{3}, \underline{10}]$, hence it is possible to compress the acquired images without losing significant information content. For the same reason, the illumination of the medium can be limited to a small number of lowfrequency illumination patterns, which results in a decrease in both acquisition and reconstruction times. The latter approach is referred to as structured illumination.

Fast reconstruction algorithms in FDOT using data compression of very large fluorescence data sets have been demonstrated on simulated data [11] and experimentally for slab phantoms [9,12]. A fundamental step toward the application of this technique to animal models relies on the multiple-view approach. This means to rotate the sample and to repeat the source-detector measurements several times $[11,13]$. It is worth mentioning that the multiple-view approach leads to an increase in the size of the data set, which represents a further argument in favor of data compression.

The aim of this work is to experimentally demonstrate, for the first time to the best of our knowledge, the use of a multiple-view approach in FDOT, using both structured illumination for the sources and data compression for the measurements. We believe that this is a fundamental step toward the in vivo application of the proposed technique. The performances, in terms of spatial resolution and computational time, are evaluated and discussed.

Here is a brief description of the experimental setup. The illumination optical pattern is produced by delivering a $\mathrm{He}-\mathrm{Ne}$ laser beam at $633 \mathrm{~nm}$ on a digital micromirror device (DMD Discovery 1100, Vialux, Germany). The pattern uploaded on the DMD is projected through a lens on a cylindrical phantom located on a motorized rotational stage. The spatially modulated light projected to the sample is generated within a rectangle $(16 \mathrm{~mm} \times 32 \mathrm{~mm})$, whose upper edge is $7 \mathrm{~mm}$ from the upper basis of the cylinder, as shown in Fig. 1(a). The fluorescence light, exiting from the opposite side of the sample, is spectrally filtered by a combination of two filters (interference filter XF 3076-Omega and high-pass filter RG-695-Schott with the cutoff wavelength at $695 \mathrm{~nm}$ ) and imaged by 


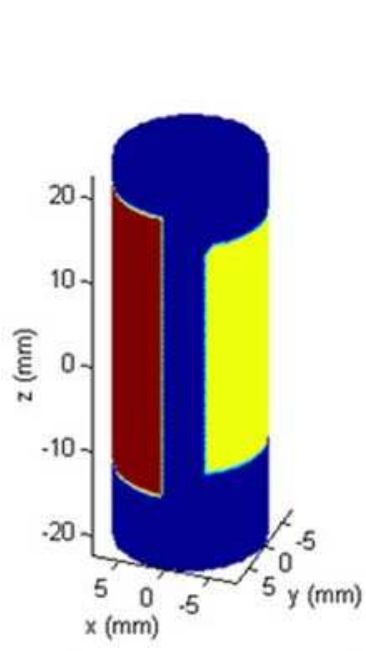

(a)

(b)
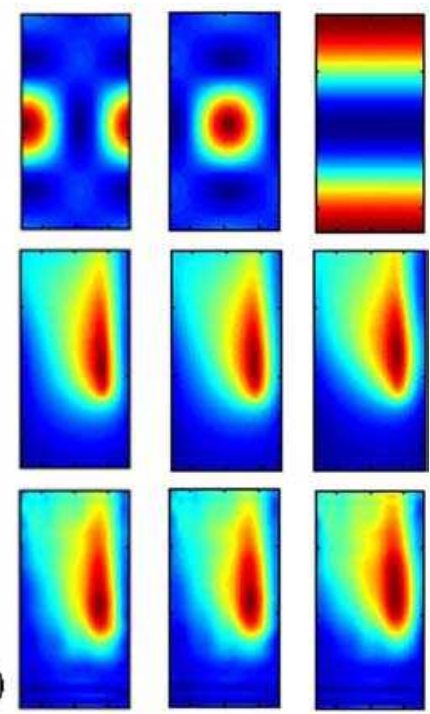

Fig. 1. (Color online) (a) Illumination (right) and detection (left) areas, (b) first row, examples of three illumination patterns; second row, Born normalized fluorescence images; third row, compressed images with $K=64$.

an objective lens $\left(f=50 \mathrm{~mm}, f^{\#}=2.8\right.$, Nikon Co., Japan $)$ on a 16 bit low noise cooled $\left(-40^{\circ}\right) \mathrm{CCD}$ (Versarray 512 , Princeton Instruments, USA). By removing the filter, the diffused light at the excitation wavelength can be acquired. The phantom is an epoxy resin cylinder $\left(\varnothing 18 \mathrm{~mm}\right.$, height of $45 \mathrm{~mm}, \mu_{a}=0.01 \mathrm{~mm}^{-1}$, and $\mu_{s}^{\prime}=$ $\left.0.82 \mathrm{~mm}^{-1}\right)$ with two cylindrical holes $(\varnothing 2 \mathrm{~mm})$ filled with a fluorescent dye (Nile Blue). The dimensions and optical parameters of the phantom have been chosen to mimic small animals. As shown in Fig. 2(a), one inclusion is parallel to the axis of the phantom while the second inclusion is oblique. Both of them are $26 \mathrm{~mm}$ long. For each source and rotation angle of the cylinder, the acquisition time is $30 \mathrm{~s}$ and $50 \mathrm{~ms}$ for the fluorescence and excitation lights, respectively. The whole experimental procedure is fully computer-controlled to speed up the measurement.

We adopted a structured illumination approach, which allows one to reduce the number of measurements while increasing the laser power that can be delivered to the sample within safety limits. The choice of wavelet scaling functions $\mathbf{s}_{j}, j \in\{1, \ldots, J\}$ as source patterns has been guided by the compression scheme [9].

The ratio between images at the fluorescence wavelength, named $\mathbf{y}^{\mathbf{m}}$, and images at the excitation wavelength, named $\mathbf{y}^{\mathbf{x}}$, is performed. The resulting images $\hat{\mathbf{y}}=\mathbf{y}^{\mathbf{m}} / \mathbf{y}^{\mathbf{x}}$, also called Born normalized images, are then wavelet-transformed using a Battle-Lemarié wavelet transform that proved to be particularly suited to fluorescence images. The $K$ largest absolute components in the transformed images are retained while the other components are discarded. This results in the compressed measurements $\tilde{\mathbf{y}}=\mathbf{Z} \hat{\mathbf{y}}$, where the rows of matrix $\mathbf{Z}$ contain the retained wavelet detection patterns $\mathbf{d}_{k}, k \in\{1, \ldots, K\}$.

Figure 1(b) shows three selected source patterns (first row), the corresponding Born normalized fluorescence images $\hat{\mathbf{y}}$ (second row) and the compressed images $\tilde{\mathbf{y}}$ retaining $K=64$ wavelet components (third row).
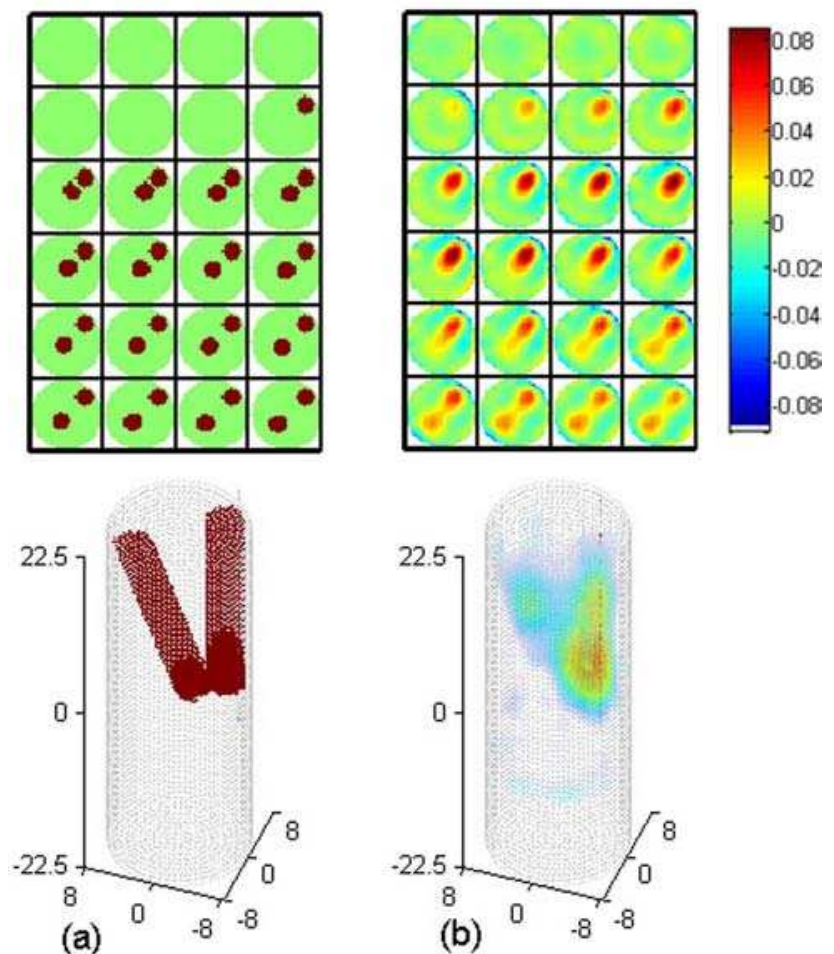

Fig. 2. (Color online) Reconstruction of the fluorochrome concentration using eight views. Top, sections at different depths at step of $1.5 \mathrm{~mm}$; bottom, 3D rendering: (a) groundtruth, (b) reconstructions.

Because our CCD camera has $512 \times 512$ pixels, the images are compressed by a factor of 4096 , with only small differences between the original and compressed images. We found that using more than $K=64$ components does not lead to any significant improvement in the reconstruction quality. The construction of the weight matrix $\mathbf{W}$, such that $\tilde{\mathbf{y}}=\mathbf{W c}$, where $\mathbf{c}$ is the fluorochrome concentration vector, has been described in detail in [9]. Here, the size of the weight matrix is $J K L \times N$, where $L$ is the number of views and $N$ is the number of voxels of the reconstruction. The weight matrix is calculated from the knowledge of the $J$ source patterns, $\mathbf{s}_{j}$, and the $K$ retained detection patterns, $\mathbf{d}_{k}$, for any of the $L$ views of the phantom. The source and detection patterns are mapped from the DMD to the cylinder surface using an orthographic projection. The projected patterns serve as source terms in the diffusion equation, which is solved by means of the finite element method [14]. The direct and adjoint densities, resulting from the use of the projected source and detection patterns, are mapped from the unstructured mesh onto a regular grid that consists of $N$ voxels.

The reconstructed fluorochrome concentration $\mathbf{c}^{*}$ is calculated as the underdetermined Tikhonov solution:

$$
\mathbf{c}^{*}=\mathbf{W}^{T}\left(\mathbf{W} \mathbf{W}^{T}+\alpha \mathbf{I}\right)^{-1} \tilde{\mathbf{y}},
$$

where the regularization parameter $\alpha$ is set to $10^{-5} \operatorname{tr}\left(\mathbf{W} \mathbf{W}^{T}\right)$.

It is worth noting that the whole reconstruction and data acquisition procedure does not exploit any 
symmetry of the cylindrical phantom. Hence, it can be applied to samples of arbitrary shape.

Figure 2(b) shows the sections at different vertical positions and three-dimensional (3D) renderings of the reconstructed fluorochrome concentration achieved using 10 illumination patterns with eight views taken at a step of 45 degrees over the complete rotation. The structure of the phantom is shown in Fig. 2(a). The two inclusions can be clearly resolved, starting from the height of $z=4 \mathrm{~mm}$. We evaluated the distances between the centers of the reconstructed and expected inclusions at various $z$ positions, obtaining a displacement of less than $1 \mathrm{~mm}$. It is worth comparing the reconstructions obtained by using single and multiple views. Figure 3 shows the reconstructed fluorochrome concentration based on a single view by using the same illumination patterns and data compression as previously reported. Figures 3(a) and 3(b) show the reconstructions obtained with a single view in the best case and rotated 45 degrees with respect to it, respectively. It is worth noting that, with a single view, the reconstruction quality is extremely dependent on the selected view. The multipleview reconstruction shows an improvement with respect to the single view approach. In particular, the two inclusions can be discriminated at a lower depth and with higher transversal spatial resolution, as demonstrated by the almost circular shape of the reconstructed inclusions shown in Fig. 2(b). This is in agreement with the
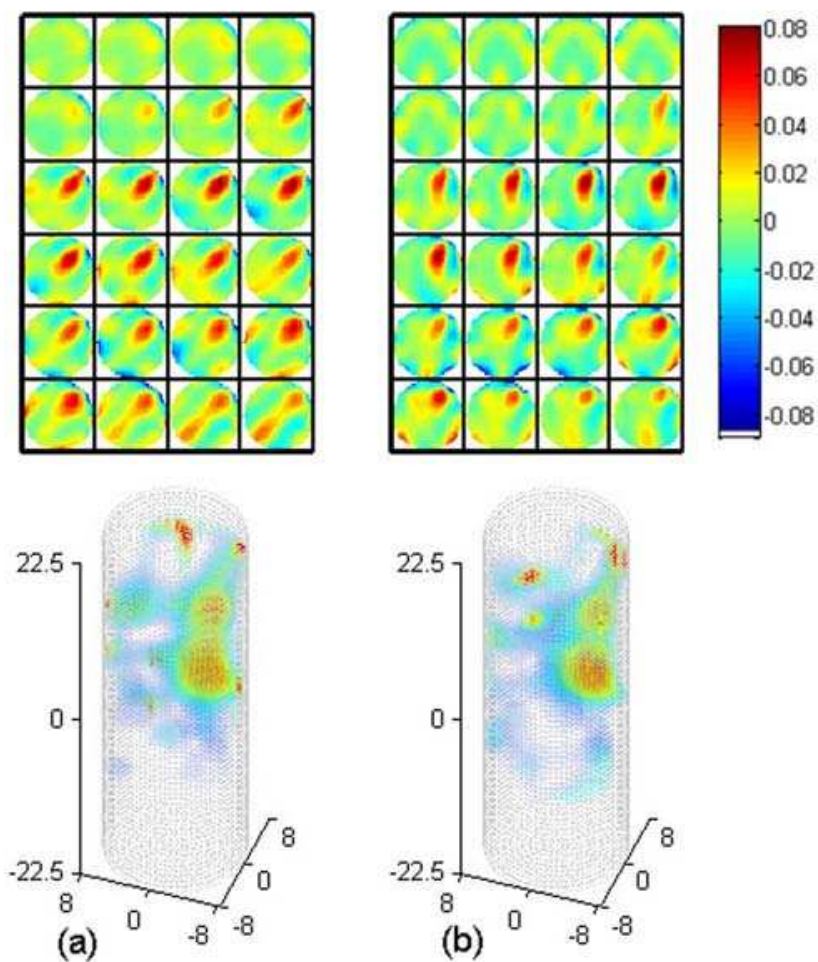

Fig. 3. (Color online) Reconstructions of the fluorochrome concentration using a single view. Top, sections at different depths at step of $1.5 \mathrm{~mm}$; bottom, 3D rendering: (a) best view, (b) best view rotated 45 degrees. results reported in [9] on slab geometry, where the transversal resolution obtained by the reconstruction with a single view is quite limited and only partially improved by the use of structured illumination. Concerning longitudinal direction, it is possible to localize the end of the inclusion with analogous performance with single and multiple views, as expected due to the symmetry of the problem. In order to quantify the overall reconstruction capability, we estimate the reconstruction error $\epsilon=$ $\left\|\mathbf{c}_{\text {true }}-\mathbf{c}\right\|^{2} /\left\|\mathbf{c}_{\text {true }}\right\|^{2}$, obtaining $\epsilon=0.71, \epsilon=0.78$, and $\epsilon=$ 0.91 for the multiple view and the two single-view cases, respectively. Finally, it is worth reporting the computational times. It took less than 5 min to calculate the weight matrix and about $10 \mathrm{~s}$ for the reconstruction, on a $2.4 \mathrm{GHz}$ and 4 Gbytes RAM personal computer.

In conclusion, in this work we have performed FDOT reconstruction by applying data compression and multiple views with patterned illumination. This strategy allowed us to drastically reduce the computational time while still obtaining high reconstruction quality. We believe that optimization of the illumination pattern [15] combined with data compression can further increase the spatial resolution of FDOT, with an additional reduction of computation and acquisition times.

This work was supported in part by the Royal Society, International Joint Project 2009/R2, and the CARIPLO Foundation (grant 2009-2626).

\section{References}

1. R. Weissleder and V. Ntziachristos, Nat. Med. 9, 123 (2003).

2. S. R. Arridge and J. C. Schotland, Inverse Probl. 25, 123010 (2009).

3. D. J. Cuccia, F. Bevilacqua, A. J. Durkin, and B. J. Tromberg, Opt. Lett. 30, 1354 (2005).

4. S. D. Konecky, A. Mazhar, D. Cuccia, A. J. Durkin, J. C. Schotland, and B. J. Tromberg, Opt. Express 17, 14780 (2009).

5. C. D'Andrea, N. Ducros, A. Bassi, S. Arridge, and G. Valentini, Biomed. Opt. Express 1, 106 (2010).

6. A. Joshi, W. Bangerth, and E. M. Sevick-Muraca, Opt. Express 14, 6516 (2006).

7. J. Ripoll, Opt. Lett. 35, 688 (2010).

8. V. Venugopal, J. Chen, F. Lesage, and X. Intes, Opt. Lett. 35, 3189 (2010).

9. N. Ducros, C. D'Andrea, G. Valentini, T. Rudge, S. R. Arridge, and A. Bassi, Opt. Lett. 35, 3676 (2010).

10. V. A. Markel and J. C. Schotland, Phys. Rev. E 70, 056616 (2004).

11. T. J. Rudge, V. Y. Soloviev, and S. R. Arridge, Opt. Lett. 35, 763 (2010).

12. G. Y. Panasyuk, Z. M. Wang, J. C. Schotland, and V. A. Markel, Opt. Lett. 33, 1744 (2008).

13. N. Deliolanis, T. Lasser, D. Hyde, A. Soubret, J. Ripoll, and V. Ntziachristos, Opt. Lett. 32, 382 (2007).

14. M. Schweiger and S. R. Arridge, "TOAST Image Reconstruction package," http://web4.cs.ucl.ac.uk/research/vis/toast/.

15. J. Dutta, C. Ahn, A. A. Joshi, and R. M. Leahy, Phys. Med. Biol. 55, 2961 (2010). 